Document downloaded from:

http://hdl.handle.net/10251/43380

This paper must be cited as:

Bonet Solves, JA.; Wright, JDM. (2011). Non-Commutative Locally Convex Measures. Quarterly Journal of Mathematics. 62(1):21-38. doi:10.1093/qmath/hap018.

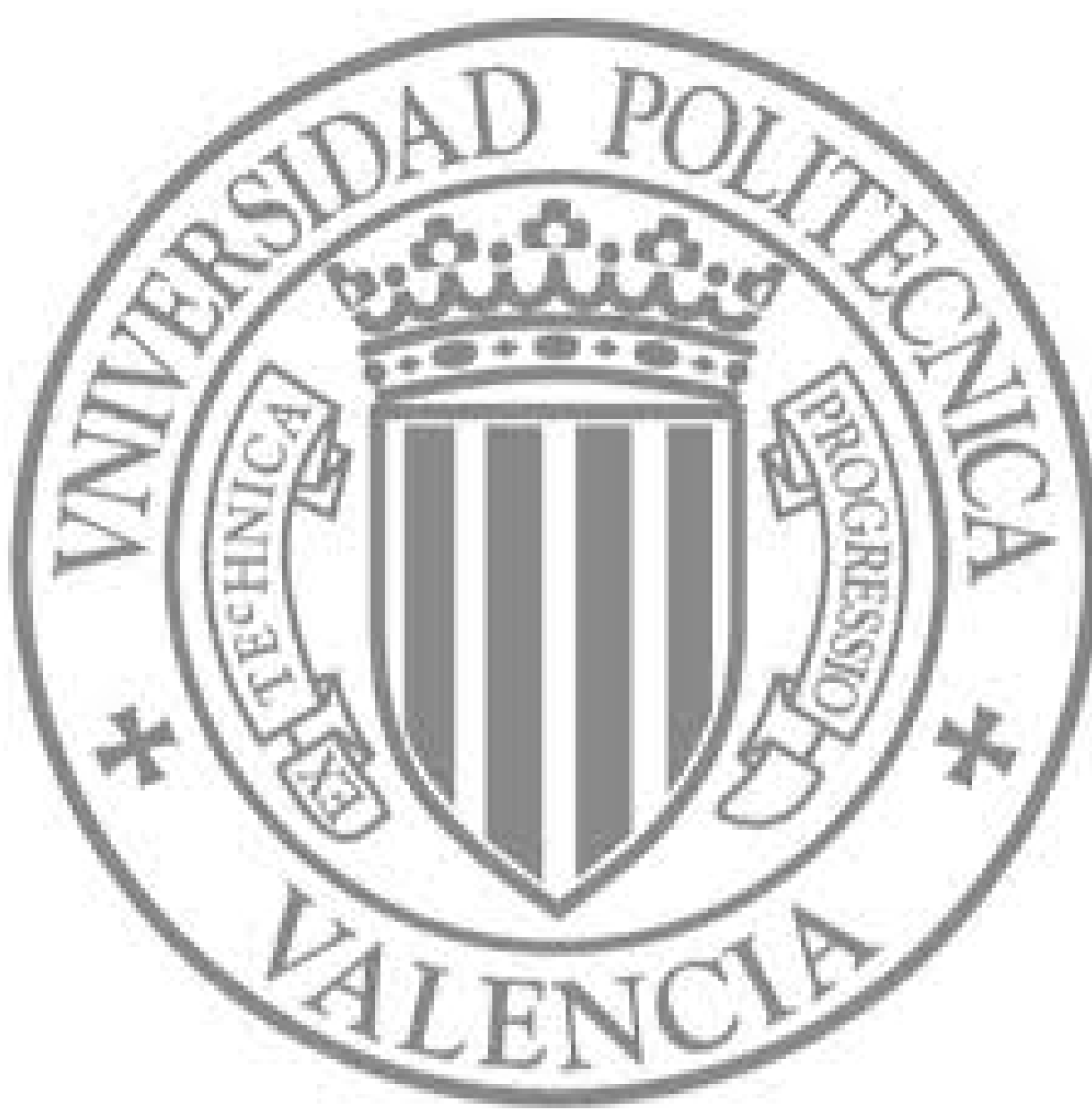

The final publication is available at

http://qjmath.oxfordjournals.org/content/62/1/21

Copyright Oxford University Press (OUP): Policy B - Oxford Open Option A 


\title{
Non-commutative locally convex measures
}

\author{
José Bonet and J. D. Maitland Wright
}

\begin{abstract}
We study weakly compact operators from a $C^{*}$-algebra with values in a complete locally convex space. They constitute a natural non-commutative generalization of finitely additive vector measures with values in a locally convex space. Several results of Brooks, Saîto and Wright are extended to this more general setting. Building on an approach due to Saîto and Wright, we obtain our theorems on non-commutative finitely additive measures with values in a locally convex space, from more general results on weakly compact operators defined on Banach spaces $X$ whose strong dual $X^{\prime}$ is weakly sequentially complete. Weakly compact operators are also characterized by a continuity property for a certain "Right topology" as in joint work by Peralta, Villanueva, Wright and Ylinen.
\end{abstract}

\section{Introduction}

In 1970, D. R. Lewis [19], extending previous work of Bartle, Dunford and Schwartz, and of Grothendieck, proved the following result (See also [17]). Let $K$ be a compact Hausdorff space, let $E$ be a complete locally convex space and let $T \in L(C(K), E)$ a continuous operator. $T$ is weakly compact if and only if there is a regular measure $\mu: \Sigma \rightarrow E$ on the Borel subsets of $K$ such that

$$
T(f)=\int_{K} f(t) \mu(d t) \quad \text { for each } f \in C(K) .
$$

A linear operator $T \in L(X, Y)$ between Banach spaces is weakly compact if it maps the closed unit ball of $X$ into a weakly relatively compact subset of $Y$. There are two possible extensions of this concept when the continuous linear operator $T \in L(F, E)$ is defined between locally convex spaces $F$ and $E$. As in [10], we say that $T$ is reflexive if it maps bounded sets into weakly relatively compact sets, and it is called weakly compact (as in $[18,42.2]$ ) if there is a 0-neighborhood $U$ in $F$ such that $T(U)$ is weakly relatively compact in $E$. It can be easily seen that if $T \in L(F, E)$ is weakly compact, then $T$ is reflexive. Although the converse is true if $F$ is a Banach space, in general this is false, as the identity $T: E \rightarrow E$ on an infinite dimensional Fréchet Montel space $E$ shows. One can take, for example, the space $E$ of entire functions on the complex plane endowed with the compact open topology.

2000 Mathematics Subject Classification. Primary: 47B07, secondary: 46L05, 46A03, 46A04, 47B48, $46 \mathrm{~L} 51$.

Key words and phrases. Weakly compact operators, $C^{*}$-algebras, locally convex spaces, vector measures, Fréchet spaces, Right topology.

The research of Bonet was partially supported by MEC and FEDER Project MTM2007-62643 and by GV Project Prometeo/2008/101. The support of the University of Aberdeen and the Universidad Politécnica of Valencia is gratefully acknowledged. 
From the point of view of the articles $[5,6,7,38]$, the natural non-commutative generalization of a (finitely additive) vector measure with values in a Banach space is a weakly compact operator from a $C^{*}$-algebra with values in a Banach space. The purpose of this paper is to study weakly compact operators $T: A \rightarrow E$ from a $C^{*}$-algebra $A$ into a complete locally convex space $E$. They constitute the natural non-commutative version of a vector measure with values in $E$. As in the paper by Saitô and Wright [33], we obtain our results on non-commutative finitely additive measures with values in a locally convex space, from more abstract results on weakly compact operators defined on Banach spaces $X$ whose strong dual $X^{\prime}$ is weakly sequentially complete. Weakly compact operators $T: X: \rightarrow E$ in our setting are also characterized by a continuity property for a certain "Right topology" $\rho(X)$ in $X$, as in [27]. A recent expository article on this topic is [40].

We obtain extensions of results of Brooks, Saitô and Wright [5, 6, 7, 8, 9, 38] from the case where $E$ is a Banach space. We generalize results of Lewis [19] and Panchapagesan [23, 24] from commutative to non-commutative $C^{*}$-algebras.

We also extend an elegant result of Akemann, Dodds and Gamlen [2] by showing that a continuous linear map $T$ from a $C^{*}$-algebra into a complete locally convex space which contains no copy of $c_{0}$ must be weakly compact.

\section{Notation and preliminaries}

In this article $A$ stands for a $C^{*}$-algebra, $A_{1}$ for its closed unit ball, $A^{\prime}$ for the dual of $A$ and $A^{\prime \prime}$ for the second dual of $A$. The space $A^{\prime \prime}$ can be identified with the von Neumann envelope of $A$ in its universal representation. This and other standard results on operator algebras may be found in $[22,25,34]$. Throughout this paper let $M$ denote a von Neumann algebra with predual $M_{*}$. As usual we do not distinguish between normal functionals on $M$ and elements of $M_{*}$.

We use standard notation for functional analysis and locally convex spaces $[13,14,18$, $21,28]$. For a locally convex space $E=(E, \tau), E^{\prime}$ stands for the topological dual of $E$, we denote by $\sigma\left(E, E^{\prime}\right), \mu\left(E, E^{\prime}\right)$ and $\beta\left(E, E^{\prime}\right)$ the weak, Mackey and strong topologies on $E$ respectively. Sometimes we write $E_{b}^{\prime}$ to denote the strong dual $\left(E^{\prime}, \beta\left(E^{\prime}, E\right)\right)$ of $E$. The family of all absolutely convex 0-neighborhoods of a locally convex space $E$ is denoted by $\mathcal{U}_{0}(E)$, the family of all absolutely convex bounded subsets of $E$ by $\mathcal{B}(E)$, and the family of all continuous seminorms on $E$ by $c s(E)$. If $E$ is a locally convex space and $q \in c s(E), E_{q}$ is the Banach space which appears as the completion of $(E / \operatorname{Kerq}, \hat{q}), \hat{q}(x+\operatorname{Kerq})=q(x)$, $x \in E$. We denote by $\pi_{q}: E \rightarrow E_{q}, \pi_{q}(x)=x+$ ker $q$ the canonical map. Observe that $\left\|\pi_{q}(x)\right\|_{E_{q}}=q(x)$ for all $x \in E$. If $q \in \operatorname{cs}(E)$ then $U_{q}:=\{x \in E \mid q(x) \leq 1\} \in \mathcal{U}_{0}(E)$ and it is closed. If $U \in \mathcal{U}_{0}(E)$, the Minkowski functional of $U, q_{U}(x)=\inf \{\lambda>0: x \in \lambda U\}$ is a seminorm on $E$. Moreover $\left\{x \in E \mid q_{U}(x)<1\right\} \subset U \subset\left\{x \in E \mid q_{U}(x) \leq 1\right\}$. If $B \in \mathcal{B}(E)$, the normed space generated by $B$ is $E_{B}:=\left(\operatorname{span} B, p_{B}\right), p_{B}$ being the Minkowski functional of $B$. If $B \in \mathcal{B}(E)$, then $E_{B} \hookrightarrow E$ continuously and we denote the injection by $j_{B}: E_{B} \rightarrow E$. If $E$ is complete, $E_{B}$ is a Banach space for every $B \in \mathcal{B}(E)$ which is closed. If $B \subset E$, the polar of $B$ in $E^{\prime}$ is

$$
B^{\circ}:=\left\{u \in E^{\prime}|| u(x) \mid \leq 1 \text { for all } x \in B\right\} .
$$

A subset $C \subset E^{\prime}$ is called $(E, \tau)$-equicontinuous (or simply equicontinuous) if there is 
$U \in \mathcal{U}_{0}(E)$ such that $C \subset U^{\circ}$. If $q \in \operatorname{cs}(E), U_{q} \in \mathcal{U}_{0}(E)$ and, by the Hahn Banach theorem,

$$
q(x)=\sup _{u \in U_{q}^{\circ}}|u(x)| .
$$

Moreover, $u \in U_{q}^{\circ}$ if and only if $|u(x)| \leq q(x)$ for all $x \in E$.

The space of all (continuous, linear) operators between the locally convex spaces $F$ and $E$ is denoted by $L(F, E)$, and we write $L_{b}(F, E)$ when this space is endowed with the topology of uniform convergence on the bounded subsets of $F$. A basis of 0-neighborhoods in $L_{b}(F, E)$ is given by $W(B, V):=\{f \in L(F, E) \mid f(B) \subset V\}$, as $B$ runs in $\mathcal{B}(F)$ and $V$ in $\mathcal{U}_{0}(E)$. The transpose of an operator $T$ is denoted by $T^{\prime}: E^{\prime} \rightarrow F^{\prime}$, and the bitranspose by $T^{\prime \prime}: F^{\prime \prime} \rightarrow E^{\prime \prime}$. If $X$ is a Banach space and $E$ is a locally convex space an operator $T \in L(X, E)$ is weakly compact if $T\left(X_{1}\right)$ is relatively $\sigma\left(E, E^{\prime}\right)$-compact in $E$. Here $X_{1}$ stands for the closed unit ball of $X$. We observe that $T \in L(X, E)$ is weakly compact if and only if $\pi_{q} \circ T: X \rightarrow E_{q}$ is weakly compact for each $q \in \operatorname{cs}(E)$.

Here is Grothendieck's extension of Gantmacher's Theorem; see [18, 42.2.(1)] and [12]. In fact Grothendieck's result is a characterization of reflexive operators between two locally convex spaces with $E$ complete.

Theorem 2.1 Let $X$ be a Banach space and let $E$ be a complete locally convex space. The following are equivalent for $T \in L(X, E)$

(1) $T$ is weakly compact.

(2) $T^{\prime \prime}\left(X^{\prime \prime}\right) \subset E$.

(3) For each $C \subset E^{\prime}$ which is equicontinuous, $T^{\prime}(C)$ is relatively $\sigma\left(X^{\prime}, X^{\prime \prime}\right)$-compact.

A Fréchet space is a complete metrizable locally convex space. We refer the reader to $[3,18,21]$ for the theory of Fréchet and (DF)-spaces. In the rest of the article $E$ always stands for a complete locally convex space, unless explicitly stated.

\section{Weakly compact operators from a Banach space into a lo- cally convex space}

Our first result is an extension of [33, Theorem 2.1].

Theorem 3.1 Let $A$ be a Banach space such that $\left(A^{\prime}, \sigma\left(A^{\prime}, A^{\prime \prime}\right)\right)$ is sequentially complete and let $E$ be a complete locally convex space. Let $\left(T_{n}\right)_{n}$ be a sequence of weakly compact operators from $A$ into $E$. If $\left(T_{n}^{\prime \prime} z\right)_{n}$ is a Cauchy sequence in $E$ for each $z \in A^{\prime \prime}$, then there is a weakly compact operator $T: A \rightarrow E$ such that $T_{n}^{\prime \prime} z \rightarrow T^{\prime \prime} z$ in $E$ for each $z \in A^{\prime \prime}$.

Proof. For each $a \in A^{\prime \prime}$ define $T_{0} z:=\lim _{n} T_{n}^{\prime \prime} z \in E$, which exists since $\left(T_{n}^{\prime \prime} z\right)_{n}$ is a Cauchy sequence in $E$ and $E$ is complete. By the Banach Steinhaus uniform boundedness theorem (see e.g., Chapter IV, Section 2, Corollary 1 in [31]), the linear operator $T_{0}: A^{\prime \prime} \rightarrow E$ is continuous. We denote by $T$ the restriction of $T_{0}$ to $A$. Clearly $T \in L(A, E)$.

Fix $u \in E^{\prime}$. For each $z \in A^{\prime \prime}$, we have

$$
\left\langle T_{0} z, u\right\rangle=\lim _{n}\left\langle T_{n}^{\prime \prime} z, u\right\rangle=\lim _{n}\left\langle z, T_{n}^{\prime} u\right\rangle,
$$


and the sequence $\left(T_{n}^{\prime} u\right)_{n}$ is $\sigma\left(A^{\prime}, A^{\prime \prime}\right)$-Cauchy in $A^{\prime}$ for each $u \in E^{\prime}$. By the sequential completeness of $\left(A^{\prime}, \sigma\left(A^{\prime}, A^{\prime \prime}\right)\right)$, for each $u \in E^{\prime}$ there is $\alpha(u) \in A^{\prime}$ such that $\langle z, \alpha(u)\rangle=\left\langle T_{0} z, u\right\rangle$ for each $z \in A^{\prime \prime}$.

We claim that $T_{0}:\left(A^{\prime \prime}, \sigma\left(A^{\prime \prime}, A^{\prime}\right)\right) \rightarrow\left(E, \sigma\left(E, E^{\prime}\right)\right)$ is continuous. Indeed, let $\left(z_{i}\right)_{i \in I}$ be a net in $A^{\prime \prime}$ converging to 0 for the topology $\sigma\left(A^{\prime \prime}, A^{\prime}\right)$. Given $u \in E^{\prime}$, we select $\alpha(u) \in A^{\prime}$ such that $\left\langle z_{i}, \alpha(u)\right\rangle=\left\langle T_{0} z_{i}, u\right\rangle$ for each $i \in I$. This implies $\lim _{i}\left\langle T_{0} z_{i}, u\right\rangle=\lim _{i}\left\langle z_{i}, \alpha(u)\right\rangle=0$.

The claim that we have just proved implies that the image $T_{0}\left(A_{1}^{\prime \prime}\right)$ of the unit ball $A_{1}^{\prime \prime}$ of $A^{\prime \prime}$ is $\sigma\left(E, E^{\prime}\right)$ relatively compact in $E$. Therefore $T\left(A_{1}\right)$ is also $\sigma\left(E, E^{\prime}\right)$ relatively compact in $E$, and $T: A \rightarrow E$ is weakly compact. By Grothendieck's extension of Gantmacher's theorem [18, 42.2.(1)], this implies that $T^{\prime \prime}\left(A^{\prime \prime}\right) \subset E$, which is equivalent to the continuity of the operator $T^{\prime}:\left(E^{\prime}, \sigma\left(E^{\prime}, E\right)\right) \rightarrow\left(A^{\prime}, \sigma\left(A^{\prime}, A^{\prime \prime}\right)\right)$. This, in turn, implies that $T^{\prime \prime}:\left(A^{\prime \prime}, \sigma\left(A^{\prime \prime}, A^{\prime}\right)\right) \rightarrow\left(E, \sigma\left(E, E^{\prime}\right)\right)$ is continuous.

Now $T_{0}$ and $T^{\prime \prime}$ are two continuous operators from $\left(A^{\prime \prime}, \sigma\left(A^{\prime \prime}, A^{\prime}\right)\right)$ into $\left(E, \sigma\left(E, E^{\prime}\right)\right)$ which coincide on $A$, which is a $\sigma\left(A^{\prime \prime}, A^{\prime}\right)$-dense subspace of $A^{\prime \prime}$. Thus $T^{\prime \prime}=T_{0}$, and the proof is complete.

If $A$ is a $C^{*}$-algebra, then its dual $A^{\prime}$ is the predual of a von Neumann algebra and, by [34, Corollary III.5.2], $\left(A^{\prime}, \sigma\left(A^{\prime}, A^{\prime \prime}\right)\right)$ is sequentially complete. Accordingly Theorem 3.1 can be applied for operators from a $C^{*}$-algebra into a complete locally convex space $E$. In fact, it is possible to improve the result in the case of $C^{*}$-algebras, thus extending [5, Corollary $3.3]$ which is a generalization to $C^{*}$-algebras of a theorem of Dieudonné in classical measure theory. To do this we need the following definition. A projection $p \in A^{\prime \prime}$ is said to be a range projection for $A$ if there exists $b \in A, 0 \leq b \leq 1$, such that the monotone increasing sequence $\left(b^{1 / n}\right)_{n}$ converges to $p$ in the topology $\sigma\left(A^{\prime \prime}, A^{\prime}\right)$. We write $p=R P(b)$.

The following non-commutative extension of a fundamental Theorem of Dieudonné [11] was proved by Brooks, Saitô and Wright in [5] Theorem 3.2 (Generalized Dieudonné Theorem): Let $A$ be a $C^{*}$-algebra, which is not assumed to be unital. Let $\left(\phi_{n}\right)_{n}$ be a sequence in $A^{\prime}$ such that for every range projection $p \in A^{\prime \prime}$ the limit $\lim _{n \rightarrow \infty} \phi_{n}(p)$ exists. Then there is a unique $\phi \in A^{\prime}$ such that for all $x \in A^{\prime \prime}, \phi(x)=\lim _{n \rightarrow \infty} \phi_{n}(x)$.

Lemma 3.2 Let $A$ be a $C^{*}$-algebra and let $\phi$ be a functional on $A$, whose extension to $A^{\prime \prime}$ is also denoted by $\phi$. Let $\phi(p)=0$ for each range projection $p$ in $A^{\prime \prime}$. Then $\phi$ is the zero functional.

Proof. Let $y$ be a positive self adjoint element of $A$ with $\|y\| \leq 1$. Then $\left(y^{1 / n}\right)_{n}$ increases monotonically to a range projection $e$. Then $\phi(e)=0$. Let $C_{0}$ be the closed commutative $C^{*}$-algebra generated by $y$. Let $C$ be the algebra generated by $y$ and $e$. So $C$ is unital and $C \subset A^{\prime \prime}$. Let $K \subset \mathbb{R}$ be the spectrum of $y$. Let $\pi$ be the spectral isomorphism of $C_{0}$ into $A^{\prime \prime}$. Then $\pi$ has a canonical extension to a $\sigma$-homomorphism $\pi^{\infty}$ of $B^{\infty}(K)$, the bounded Baire measurable functions on $K$, into $A^{\prime \prime}$. Let $U$ be any open subset of $K$. Then $\chi_{U}$ is lower semicontinuous. Since $K$ is metrizable, each open subset is an $F_{\sigma}$-set. So, by Uryshon's Lemma, there exists a monotone increasing sequence of positive elements of $C_{0},\left(a_{n}\right)_{n}$, which increases pointwise to $\chi_{U}$. Let $b=\sum_{n=1}^{\infty} 1 / 2^{n} a_{n}$. Then $\left(b^{1 / n}\right)_{n}$ increases pointwise to $\chi_{U}$. So $\left(\pi\left(b^{1 / n}\right)\right)_{n}$ increases strong* to $\pi^{\infty}\left(\chi_{U}\right)$. Thus $\pi^{\infty}\left(\chi_{U}\right)$ is a range projection in $A^{\prime \prime}$. So 
$\phi \pi^{\infty}\left(\chi_{U}\right)=0$. Now, let $f$ be a bounded lower semicontinuous function on $K$. Then, as remarked by Dieudonné [11], $f$ is the uniform limit of linear combinations of functions of the form $\chi_{U}$, where $U$ is open. So $\phi \pi^{\infty}(f)=0$. So $\phi$ vanishes on $\pi(C)$, in particular $\phi(y)=0$. It follows by linearity that $\phi$ vanishes on $A$.

Theorem 3.3 Let $A$ be a $C^{*}$-algebra, let $E$ be a complete locally convex space and let $\left(T_{n}\right)_{n}$ be a sequence of weakly compact operators $T_{n}: A \rightarrow E$. Suppose that, whenever $p \in A^{\prime \prime}$ is a range projection, $\lim _{n \rightarrow \infty} T_{n}^{\prime \prime}(p)$ exists in $E$. Then there is a unique weakly compact operator $T: A \rightarrow E$ such that $T^{\prime \prime}(x)=\lim _{n \rightarrow \infty} T_{n}^{\prime \prime}(x)$ for each $x \in A^{\prime \prime}$.

Proof. Suppose first that there is $x \in A^{\prime \prime}$ such that $\left(T_{n}^{\prime \prime}(x)\right)_{n} \subset E$ is not a Cauchy sequence in $E$. We find $q \in \operatorname{cs}(E)$ and an increasing sequence $(n(i))_{n}$ in $\mathbb{N}$ such that $q\left(T_{n(i+1)}^{\prime \prime}(x)-\right.$ $\left.T_{n(i)}^{\prime \prime}(x)\right)>1$ for each $i \in \mathbb{N}$. Set $S_{i}:=\left(T_{n(i+1)}-T_{n(i)}\right)^{\prime \prime}: A^{\prime \prime} \rightarrow E$, and find, for each $i \in \mathbb{N}$, $u_{i} \in E^{\prime}$ such that $\left|u_{i}(z)\right| \leq q(z)$ for all $z \in E$ and $\left|u_{i}\left(S_{i}(x)\right)\right|>1$.

We set $\phi_{i}:=\left.\left(u_{i} \circ S_{i}\right)\right|_{A} ; \phi_{i} \in A^{\prime}$ for each $i \in \mathbb{N}$. If $p \in A^{\prime \prime}$ is a range projection, by assumption, $S_{i}(p)$ converges to 0 in $E$. Hence $\left(q\left(S_{i}(p)\right)\right)_{i}$ converges to 0 , and then also $\left(\phi_{i}(p)\right)_{i}$ tends to 0 since $\left|u_{i} \circ S_{i}(p)\right| \leq q\left(S_{i}(p)\right)$. By the generalized Dieudonné theorem, there is $\phi \in A^{\prime}$ such that $\lim _{n \rightarrow \infty} \phi_{i}(b)=\phi(b)$ for each $b \in A^{\prime \prime}$. In particular, $\phi(p)=0$ for each range projection $p \in A^{\prime \prime}$. By Lemma 3.2, $\phi(y)=0$ for all $y \in A$. Then $\phi(z)=0$ for every $z \in A^{\prime \prime}$, since $\phi \in A^{\prime}$. However this contradicts $q\left(\phi_{i}(x)\right)>1$ for each $i \in \mathbb{N}$. Consequently, as $E$ is complete, for each $x \in A^{\prime \prime}, L(x):=\lim _{n \rightarrow \infty} T_{n}^{\prime \prime}(x)$ exists. The conclusion follows from Theorem 3.1, since $\left(A^{\prime}, \sigma\left(A^{\prime}, A^{\prime \prime}\right)\right)$ is sequentially complete.

\section{Extending a result of Ryan on weakly compact operators}

In this section we extend results due to Ryan [32], Ylinen [42] and to Saitô and Wright [33] about weakly compact operators from a Banach space into the space of convergent or null sequences in another Banach space.

For a locally convex space $E$, we denote by $\ell_{\infty}(E)$ the space of all bounded sequences $\left(x_{n}\right)_{n}$ in $E$, endowed with the topology of uniform convergence generated by the seminorms $P_{q}\left(\left(x_{n}\right)_{n}\right):=\sup _{n} q\left(x_{n}\right)$, for each continuous seminorm $q$ in $E$. The closed subspaces of convergent and of null sequences are denoted by $c(E)$ and $c_{0}(E)$ respectively. On the other hand, the space of all absolutely summable sequences $\left(y_{n}\right)_{n}$ in $E$ is denoted by $\ell_{1}(E)$, and it is endowed with the continuous seminorms $P_{q}^{1}\left(\left(y_{n}\right)_{n}\right):=\sum_{n} q\left(y_{n}\right)$. By [30, 1.5.8], if $E$ is a Fréchet or a (DF)-space, then for every $\left(y_{n}\right)_{n}$ in $\ell_{1}(E)$, there is an absolutely convex, closed, bounded subset $B$ of $E$ such that $\sum_{n} p_{B}\left(y_{n}\right) \leq 1$. Here $p_{B}$ is the Minkowski functional of $B$. This permits us to show, proceeding as in the proofs of [28, 4.8.7] and [3, Theorem 10], using the stability properties of (DF)-spaces [28, 8.3.60 and 11.3.14], the following result.

Proposition 4.1 Let $E$ be a Fréchet space or a complete (DF)-space, and let $j: E \rightarrow E^{\prime \prime}$ be the canonical embedding. We have the following dualities:

(1) $c(E)_{b}^{\prime} \simeq \ell_{1}\left(E_{b}^{\prime}\right)$, and the duality is given by

$$
\left\langle\left(x_{1}, x_{2}, \ldots\right),\left(u_{0}, u_{1}, u_{2}, \ldots\right)\right\rangle:=\left\langle\lim _{n} x_{n}, u_{0}\right\rangle+\sum_{n=1}^{\infty}\left\langle x_{n}, u_{n}\right\rangle .
$$


(2) $\ell_{1}\left(E_{b}^{\prime}\right)_{b}^{\prime} \simeq \ell_{\infty}\left(E_{b}^{\prime \prime}\right)$, the duality is given by

$$
\left\langle\left(u_{0}, u_{1}, u_{2}, \ldots\right),\left(z_{0}, z_{1}, z_{2}, \ldots\right)\right\rangle:=\sum_{n=0}^{\infty}\left\langle u_{n}, z_{n}\right\rangle .
$$

Furthermore the embedding $J: c(E) \rightarrow \ell_{\infty}\left(E_{b}^{\prime \prime}\right)$ is given by

$$
J\left(\left(x_{1}, x_{2}, \cdots\right)\right):=\left(j\left(\lim _{n} x_{n}\right), j\left(x_{1}\right), j\left(x_{2}\right), \cdots\right) .
$$

Lemma 4.2 Let $A$ be a Banach space and $E$ a complete locally convex space. Let $\left(T_{n}\right) \subset$ $L(A, E)$ be a sequence of operators such that $T_{\infty} a:=\lim _{n} T_{n} a$ exist in $E$ for each $a \in A$. Then

(1) The linear map $T_{\infty}: A \rightarrow E$ defined by $T_{\infty} a:=\lim _{n} T_{n} a, a \in A$, is continuous.

(2) The operator $\tilde{T}: A \rightarrow c(E), \tilde{T} a:=\left(T_{n} a\right)_{n}, a \in A$, is well defined and continuous.

Proof. (1) By the uniform boundedness principle (see e.g., Chapter IV, Section 2, Corollary 1 in [31]), $T_{\infty}: A \rightarrow E$ is continuous.

(2) Applying again the uniform boundedness principle (see e.g., Chapter IV, Section 2, Theorem 3 in [31]), the sequence $\left(T_{n}\right)_{n}$ is equicontinuous in $L(A, E)$. Hence, for every continuous seminorm $p$ on $E$, there is $M>0$ such that $p\left(T_{n}(x)\right) \leq M\|x\|$ for each $n \in \mathbb{N}$ and each $x \in A$. This implies that $\tilde{T}: A \rightarrow c(E)$ is continuous.

Observe that every continuous linear operator from $A$ to $c(E)$ arises in the way described in Lema 4.2 from a sequence of $\left(T_{n}\right)_{n}$ continuous linear operators from $A$ into $E$.

Lemma 4.3 Let $A$ be a Banach space and let $E$ be a Fréchet space or a complete (DF)-space. Let $\left(T_{n}\right) \subset L(A, E)$ be a sequence of operators such that $T_{\infty} a:=\lim _{n} T_{n}$ a exist in $E$ for each $a \in A$. Let $\tilde{T} a:=\left(T_{n} a\right)_{n}, a \in A$. Then, for each $U=\left(u_{0}, u_{1}, u_{2}, \ldots\right) \in c(E)^{\prime}=\ell_{1}\left(E_{b}^{\prime}\right)$ and for each $z \in A^{\prime \prime}$, we have

$$
\left\langle\tilde{T}^{\prime \prime} z, U\right\rangle=\left\langle T_{\infty}^{\prime \prime} z, u_{0}\right\rangle+\sum_{n=1}^{\infty}\left\langle T_{n}^{\prime \prime} z, u_{n}\right\rangle .
$$

Proof. For each $a \in A$ we have, according to the identifications explained above,

$$
\langle\tilde{T} a, U\rangle=\left\langle T_{\infty} a, u_{0}\right\rangle+\sum_{n=1}^{\infty}\left\langle T_{n} a, u_{n}\right\rangle
$$

Given $z \in A_{1}^{\prime \prime}$, we find a net $\left(a_{i}\right)_{i \in I} \subset A_{1}$ converging to $z$ for the topology $\sigma\left(A^{\prime \prime}, A^{\prime}\right)$. This implies that the net $\left(\tilde{T}^{\prime \prime} a_{i}\right)_{i \in I}$ converges to $\tilde{T}^{\prime \prime} z$ in the space $\left(c(E)^{\prime \prime}, \sigma\left(c(E)^{\prime \prime}, c(E)^{\prime}\right)\right)$. Moreover, for each $N \in \mathbb{N}$, the net

$$
\left\langle T_{\infty} a_{i}, u_{0}\right\rangle+\sum_{n=1}^{N}\left\langle T_{n} a_{i}, u_{n}\right\rangle, \quad i \in I
$$

converges to

$$
\left\langle T_{\infty}^{\prime \prime} z, u_{0}\right\rangle+\sum_{n=1}^{N}\left\langle T_{n}^{\prime \prime} z, u_{n}\right\rangle
$$


Since $U=\left(u_{0}, u_{1}, u_{2}, \ldots\right) \in \ell_{1}\left(E^{\prime}\right)$, and $E_{b}^{\prime}$ is either a complete (DF)-space (if $E$ is Fréchet) or a Fréchet space (if $E$ is a (DF)-space), we can find an absolutely convex closed bounded subset $B$ of $E_{b}^{\prime}$ such that $\sum_{n} p_{B}\left(u_{n}\right) \leq 1 ; p_{B}$ being the Minkowski functional of $B$. We denote by $C$ the closed absolutely convex hull in $E$ of the set $\bigcup_{n=1}^{\infty}\left(T_{n}\left(A_{1}\right) \cup T_{\infty}\left(A_{1}\right)\right)$. By the uniform boundedness theorem, the set $\left\{T_{n} \mid n \in \mathbb{N}\right\} \cup\left\{T_{\infty}\right\}$ is equicontinuous in $L(A, E)$, hence $C$ is bounded in $E$. Furthermore, since $A_{1}^{\prime \prime}$ coincides with the closure of $A_{1}$ in $\left(A^{\prime \prime}, \sigma\left(A^{\prime \prime}, A^{\prime}\right)\right.$ ), we conclude that $\bigcup_{n=1}^{\infty}\left(T_{n}^{\prime \prime}\left(A_{1}^{\prime \prime}\right) \cup T_{\infty}^{\prime \prime}\left(A_{1}^{\prime \prime}\right)\right.$ is contained in the $\sigma\left(E^{\prime \prime}, E^{\prime}\right)$-closure $D$ of $C$ in $E^{\prime \prime}$. Since $D$ is bounded and $E_{b}^{\prime \prime}$ and $B$ is bounded in $E_{b}^{\prime}$, we can find $d>0$ such that $|\langle w, v\rangle| \leq d$ for each $w \in D, v \in B$. In particular, for each $z \in A_{1}^{\prime \prime}$ and each $n \in \mathbb{N},\left|\left\langle T_{n}^{\prime \prime} z, u_{n}\right\rangle\right| \leq d p_{B}\left(u_{n}\right)$.

Fix $\varepsilon>0$. Find $N \in \mathbb{N}$ such that $d \sum_{n=N+1}^{\infty} p_{B}\left(u_{n}\right)<\varepsilon / 4$. Given $z \in A_{1}^{\prime \prime}$, select $i_{0} \in I$ such that, for $i \geq i_{0}$,

$$
\left|\left\langle\tilde{T}^{\prime \prime} z, U\right\rangle-\left\langle\tilde{T} a_{i}, U\right\rangle\right|<\varepsilon / 4
$$

and

$$
\left|\left\langle T_{\infty} a_{i}, u_{0}\right\rangle+\sum_{n=1}^{N}\left\langle T a_{i}, u_{n}\right\rangle-\left\langle T_{\infty}^{\prime \prime} z, u_{0}\right\rangle-\sum_{n=1}^{N}\left\langle T_{n}^{\prime \prime} z, u_{n}\right\rangle\right|<\varepsilon / 4 .
$$

For all $M>N$ we have

$$
\sum_{n=M+1}^{\infty}\left|\left\langle T_{n}^{\prime \prime} z, u_{n}\right\rangle\right| \leq d \sum_{n=N+1}^{\infty} p_{B}\left(u_{n}\right)<\varepsilon / 4 .
$$

Hence, the inequalities above permit us to conclude, for each $M>N$,

$$
\left|\left\langle\tilde{T}^{\prime \prime} z, U\right\rangle-\left\langle T_{\infty}^{\prime \prime} z, u_{0}\right\rangle-\sum_{n=1}^{M}\left\langle T_{n}^{\prime \prime} z, u_{n}\right\rangle\right|<\varepsilon
$$

This implies

$$
\left\langle\tilde{T}^{\prime \prime} z, U\right\rangle=\left\langle T_{\infty}^{\prime \prime} z, u_{0}\right\rangle+\sum_{n=1}^{\infty}\left\langle T_{n}^{\prime \prime} z, u_{n}\right\rangle
$$

for each $z \in A_{1}^{\prime \prime}$, and the conclusion follows.

When the identification of $c(E)^{\prime \prime}$ and $\ell_{\infty}\left(E^{\prime \prime}\right)$ is made appropriately, we have the following direct consequence of Lemma 4.3.

Corollary 4.4 Let $A$ be a Banach space and let $E$ be a Fréchet space or a complete (DF)space. Let $\left(T_{n}\right) \subset L(A, E)$ be a sequence of operators such that $T_{\infty} a:=\lim _{n} T_{n} a$ exist in $E$ for each $a \in A$. Let $\tilde{T} a:=\left(T_{n} a\right)_{n}, a \in A$. Then the bitranspose operator $\tilde{T}^{\prime \prime}: A^{\prime \prime} \rightarrow \ell_{\infty}\left(E_{b}^{\prime \prime}\right)$ satisfies $\tilde{T}^{\prime \prime} z=\left(T_{\infty}^{\prime \prime} z, T_{1}^{\prime \prime} z, T_{2}^{\prime \prime} z, \ldots\right)$.

Lemma 4.5 Let $A$ be a Banach space and let $E$ be a Fréchet space or a complete (DF)-space. Let $\tilde{T} \in L(A, c(E))$ be a weakly compact operator and let $T_{n}$ be the operators from $A$ into $E$ such that $\tilde{T} a=\left(T_{n} a\right)_{n}, a \in A$. Then each $T_{n}$ and the pointwise limit $T_{\infty}$ of $\left(T_{n}\right)_{n}$ are weakly compact. Furthermore, $T_{\infty}^{\prime \prime} z=\lim _{n} T_{n}^{\prime \prime} z$ for each $z \in A^{\prime \prime}$.

Proof. Since the canonical maps $p_{m}: c(E) \rightarrow E, p_{m}\left(\left(x_{n}\right)_{n}\right):=x_{m}$, and $p_{\infty}: c(E) \rightarrow$ $E, p_{\infty}\left(\left(x_{n}\right)_{n}\right):=\lim _{n} x_{n}$, are continuous, the weak compactness of $\tilde{T}$ implies that $T_{n}=$ $p_{n} \circ \tilde{T}, n \in \mathbb{N}$, and $T_{\infty}=p_{\infty} \circ \tilde{T}$ are weakly compact. Moreover, $\tilde{T}^{\prime \prime}\left(A^{\prime \prime}\right)$ is contained in the 
canonical image of $c(E)$ in $\ell_{\infty}\left(E^{\prime \prime}\right)$. Therefore, for each $z \in A^{\prime \prime}$ there is $x=\left(x_{k}\right)_{k} \in c(E)$ such that, for each $u \in E^{\prime},\left\langle x_{k}, u\right\rangle=\left\langle u, T_{k}^{\prime \prime} z\right\rangle$ for each $k \in \mathbb{N}$ and $\left\langle\lim _{k} x_{k}, u\right\rangle=\left\langle u, T_{\infty}^{\prime \prime}\right\rangle$. That is, $j\left(x_{n}\right)=T_{n}^{\prime \prime} z$ for all $n \in \mathbb{N}$ and $\lim _{n} j\left(x_{n}\right)=T_{\infty}^{\prime \prime} z$. In particular, $T_{\infty}^{\prime \prime} z=\lim _{n} T_{n}^{\prime \prime} z$.

Here is the extension of Ylinen [42, Corollary 2.3].

Proposition 4.6 Let $A$ be a Banach space and let $E$ be a Fréchet space or a complete (DF)space and let $\tilde{T} \in L(A, c(E))$ be an operator. Let $T_{n}$ be the operators from $A$ into $E$ such that $\tilde{T} a=\left(T_{n} a\right)_{n}, a \in A$ and let $T_{\infty}$ be the pointwise limit of $\left(T_{n}\right)_{n}$. The operator $\tilde{T}$ is weakly compact if and only if the following conditions are satisfied.

(1) $T_{n}$ is weakly compact for each $n$.

(2) For each $z \in A^{\prime \prime}, T_{\infty}^{\prime \prime} z=\lim _{n} T_{n}^{\prime \prime} z$.

(3) The operator $T_{\infty}$ is weakly compact.

Proof. By Lemma 4.5, when $\tilde{T}$ is weakly compact the three conditions are satisfied. Conversely, suppose that the conditions hold. Conditions (i) and (iii) imply that $T_{n}\left(A^{\prime \prime}\right)$ and $T_{\infty}\left(A^{\prime \prime}\right)$ are contained in $E$ for each $n$. Hence, by condition (ii), $\left(T_{\infty}^{\prime \prime} z, T_{1}^{\prime \prime} z, T_{2}^{\prime \prime} z, \ldots\right)$ belongs to the canonical image of $c(E)$ in $c(E)^{\prime \prime}$ for each $z \in A^{\prime \prime}$. By Corollary 4.4, $\tilde{T}^{\prime \prime}$ maps $A^{\prime \prime}$ into $c(E)$, and the operator $\tilde{T}$ is weakly compact.

As a consequence we obtain the following extension of a useful lemma due to Ryan [32].

Corollary 4.7 Let $A$ be a Banach space and let $E$ be a Fréchet space or a complete (DF)space. Let $T_{n}$ be the operators from $A$ into $E$ such that, for each $a \in A, \lim _{n} T_{n} a=0$ in $E$. Then the operator $\tilde{T}: A \rightarrow c_{0}(E), \tilde{T} a:=\left(T_{n} a\right)_{n}, a \in A$ is weakly compact if and only if each $T_{n}$ is weakly compact and $\lim _{n} T_{n}^{\prime \prime} z=0$ for each $z \in A^{\prime \prime}$. Furthermore, if $\tilde{T}$ is weakly compact, then $\tilde{T}^{\prime \prime} z=\left(T_{n}^{\prime \prime} z\right)_{n}$ for each $z \in A^{\prime \prime}$.

Theorem 4.8 Let $A$ be a Banach space such that $\left(A^{\prime}, \sigma\left(A^{\prime}, A^{\prime \prime}\right)\right)$ is sequentially complete and let $E$ be a Fréchet space or a complete $(D F)$-space. Let $\left(T_{n}\right)_{n}$ be a sequence of weakly compact operators from $A$ into $E$, such that $\left(T_{n}^{\prime \prime} z\right)_{n}$ is a Cauchy sequence in $E$ for each $z \in A^{\prime \prime}$. Then $\tilde{T}: A \rightarrow c(E), \tilde{T} a:=\left(T_{n} a\right)_{n}, a \in A$, is a weakly compact operator.

Proof. Since $\left(A^{\prime}, \sigma\left(A^{\prime}, A^{\prime \prime}\right)\right)$ is sequentially complete, we can apply Theorem 3.1 to find a weakly compact operator $T_{\infty} \in L(A, E)$ such that $T_{\infty}^{\prime \prime} z=\lim _{n} T_{n}^{\prime \prime} z$ for each $z \in A^{\prime \prime}$. So conditions (i)-(iii) of Proposition 4.6 are satisfied. The conclusion follows.

If the assumption that $A$ has a weakly sequentially complete dual is removed in Theorem 4.8 , the conclusion is no longer valid, as the example constructed by Ylinen in [42, Proposition $2.1]$ shows.

\section{The Right topology. A continuity characterization of reflex- ive operators between locally convex spaces.}

Let $E$ be a locally convex space. As in the work of Peralta, Villanueva, Wright and Ylinen [27], the Right topology $\rho(E)$ is the topology induced in $E$ by the Mackey topology $\mu\left(E^{\prime \prime}, E^{\prime}\right)$ of the 
dual pair $\left(E^{\prime \prime}, E^{\prime}\right)$. Recall that $\mu\left(E^{\prime \prime}, E^{\prime}\right)$ is the topology on $E^{\prime \prime}$ of the uniform convergence on the absolutely convex $\sigma\left(E^{\prime}, E^{\prime \prime}\right)$-compact subsets of $E^{\prime}$. See also Peralta's paper [26] for the Right topology on Banach spaces. The existence of the Right topology can be obtained as a consequence of the operator ideals approach in the articles of Wong [36] and [37]. Clearly $\rho(E)$ is finer than the weak topology $\sigma\left(E, E^{\prime}\right)$. Moreover, if $E$ is a barrelled space, then $\rho(E)$ is coarser than the original topology of $E$, since every absolutely convex $\sigma\left(E^{\prime}, E^{\prime \prime}\right)$-compact subset of $E^{\prime}$ is $E$-equicontinuous in this case. Accordingly, if $E$ is barrelled, the Right topology $\rho(E)$ is a topology of the dual pair $\left(E, E^{\prime}\right)$. Moreover, if $(E, \tau)$ is reflexive, then the Right topology $\rho(E)$ coincides with the original topology $\tau$ of $E$. Indeed, since $E$ is barrelled, $\tau=\mu\left(E, E^{\prime}\right)$ and $E=E^{\prime \prime}$ as $E$ is semireflexive. This implies $\mu\left(E^{\prime \prime}, E^{\prime}\right)=\tau$.

Recall that a continuous linear operator $T \in L(F, E)$ between the locally convex spaces $F$ and $E$ is called reflexive if the image of every bounded subset of $F$ is relatively $\sigma\left(E, E^{\prime}\right)$ compact in $E$. If $F$ is a normed space, an operator $T \in L(F, E)$ is reflexive if and only if it is weakly compact.

Proposition 5.1 If the continuous linear operator $T$ between the locally convex spaces $F$ and $E$ is reflexive, then $T:(F, \rho(F)) \rightarrow E$ is continuous.

Proof. If $T$ is reflexive, then $T^{\prime \prime}\left(F^{\prime \prime}\right) \subset E$ by [18, 42.2.(1)]. This implies that $T^{\prime}$ : $\left(E^{\prime}, \sigma\left(E^{\prime}, E\right)\right) \rightarrow\left(F^{\prime}, \sigma\left(F^{\prime}, F^{\prime \prime}\right)\right)$ is continuous. Therefore $T^{\prime}\left(V^{\circ}\right)$ is $\sigma\left(F^{\prime}, F^{\prime \prime}\right)$-compact in $F^{\prime}$ for each $V \in \mathcal{U}_{0}(E)$. This implies that the operator $T^{\prime \prime}:\left(F^{\prime \prime}, \mu\left(F^{\prime \prime}, F^{\prime}\right)\right) \rightarrow E$ is continuous. Taking the restriction of the topology $\mu\left(F^{\prime \prime}, F^{\prime}\right)$ to $F$, we conclude that $T:(F, \rho(F)) \rightarrow E$ is also continuous.

Proposition 5.2 Let $T \in L(F, E)$ be a continuous linear operator between locally convex spaces. Suppose that $F$ is barrelled and $E$ is complete. Assume that there is an absolutely convex neighbourhood $V \in \mathcal{U}_{0}(F)$ such that the restriction $T \mid V$ of $T$ from $V$, equipped with the topology induced by the Right topology $\rho(F)$, into $E$ is continuous. Then $T: F \rightarrow E$ is reflexive.

Proof. First observe that $T^{\prime \prime}:\left(F^{\prime \prime}, \sigma\left(F^{\prime \prime}, F^{\prime}\right)\right) \rightarrow\left(E^{\prime \prime}, \sigma\left(E^{\prime \prime}, E^{\prime}\right)\right)$ is continuous since $T \in$ $L(F, E)$. Fix $z \in F^{\prime \prime}$. There is a bounded absolutely convex subset $B$ in $F$ such that $z$ belongs to the closure $C$ of $B$ is $\left(F^{\prime \prime}, \sigma\left(F^{\prime \prime}, F^{\prime}\right)\right)$. Since $\mu\left(F^{\prime \prime}, F^{\prime}\right)$ is a topology of the dual pair $\left(F^{\prime \prime}, F^{\prime}\right)$ we can find a net $\left(x_{\lambda}\right)_{\lambda} \subset B$, which converges to $z$ for the topology $\mu\left(F^{\prime \prime}, F^{\prime}\right)$, hence the net $\left(x_{\lambda}\right)_{\lambda}$ is a Cauchy net in $(F, \rho(F))$. Since $B$ is bounded, we can find $M>0$ such that $x_{\lambda} \in M V$ for each $\lambda$. As $E$ is complete, we can apply the continuity of $T$ on $V$ endowed with the Right topology, to conclude that the net $\left(T x_{\lambda}\right)_{\lambda}$ converges to a element $y$ in $E$. On the other hand, as $\left(x_{\lambda}\right)_{\lambda}$ converges to $z$ for the topology $\sigma\left(F^{\prime \prime}, F^{\prime}\right)$, the net $\left(T x_{\lambda}\right)_{\lambda}$ converges to $T^{\prime \prime} z$ in $\left(E^{\prime \prime}, \sigma\left(E^{\prime \prime}, E^{\prime}\right)\right)$. This implies $T^{\prime \prime} z=y \in E$. Therefore $T^{\prime \prime}\left(F^{\prime \prime}\right) \subset E$, and we can apply $[18,42.2$.(1)] to conclude that $T$ is reflexive.

Theorem 5.3 Let $F$ and $E$ be complete, barrelled locally convex spaces. Let $T \in L(F, E)$ be a continuous linear operator. The following conditions are equivalent.

(1) $T$ is reflexive.

(2) $T^{\prime \prime}:\left(F^{\prime \prime}, \mu\left(F^{\prime \prime}, F^{\prime}\right)\right) \rightarrow\left(E^{\prime \prime}, \beta\left(E^{\prime \prime}, E^{\prime}\right)\right)$ is continuous. 
(3) $T:(F, \rho(F)) \rightarrow E$ is continuous.

(4) There is an absolutely convex neighbourhood $V \in \mathcal{U}_{0}(F)$ such that the restriction $T \mid V$ of $T$ from $V$, equipped with the topology induced by the Right topology $\rho(F)$, into $E$ is continuous.

Proof. The equivalence of conditions (1) and (2) follows from Grothendieck's extension of Gantmacher's theorem $\left[18,42.2\right.$.(1)] since (2) holds if and only if the image of every $\beta\left(E^{\prime}, E\right)$ bounded subset of $F^{\prime}$ is relatively $\sigma\left(F^{\prime}, F^{\prime \prime}\right)$-compact, and every $\beta\left(E^{\prime}, E\right)$ bounded set in $E^{\prime}$ is E-equicontinuous. Condition (1) implies (3) by Proposition 5.1, and (3) clearly implies (4). Finally condition (4) implies (1) by Proposition 5.2.

Proposition 5.4 Let $T: F \rightarrow E$ be a linear operator between complete, barrelled locally convex spaces. Then $T$ is continuous from $F$ to $E$ with their original topologies if and only if $T:(F, \rho(F)) \rightarrow(E, \rho(E))$ is continuous.

Proof. Assume first that $T:(F, \rho(F)) \rightarrow(E, \rho(E))$ is continuous. Since $F$ and $E$ are barrelled, the Right topologies are topologies of the dual pair $\left(F, F^{\prime}\right)$ and $\left(E, E^{\prime}\right)$ respectively. This implies that $T:\left(F, \sigma\left(F, F^{\prime}\right)\right) \rightarrow\left(E, \sigma\left(E, E^{\prime}\right)\right)$ is continuous, hence $T:\left(F, \mu\left(F, F^{\prime}\right)\right) \rightarrow$ $\left(E, \mu\left(E, E^{\prime}\right)\right)$ is continuous. Since both spaces are barrelled, their topologies coincide with the corresponding Mackey topologies. Conversely, if $T$ is continuous from $F$ to $E$, then $T^{\prime \prime}:\left(F^{\prime \prime}, \mu\left(F^{\prime \prime}, F^{\prime}\right)\right) \rightarrow\left(E^{\prime \prime}, \mu\left(E^{\prime \prime}, E^{\prime}\right)\right)$ is continuous. Passing to the induced topologies in $F$ and $E$, we conclude that $T$ is Right-Right continuous.

A continuous linear operator $T$ between locally convex spaces $F$ and $E$ is called pseudo reflexive if $T:(F, \rho(F)) \rightarrow E$ is sequentially continuous. In case $F$ is a normed space, we say that $T$ is pseudo weakly compact as in [27]. By Theorem 5.3, every reflexive operator is pseudo reflexive. As remarked in Example 8 of [27] the identity operator on $\ell_{1}$ is pseudo weakly compact but not weakly compact. As we see in Section 6 , a continuous linear operator $T: A \rightarrow E$ from a $C^{*}$-algebra into a complete locally convex space $E$ is weakly compact if and only if it is pseudo weakly compact.

Remark 5.5 Peralta, Villanueva, Wright and Ylinen proved, see e.g. Theorem 5.3 of [40], that a fundamental system of seminorms of the Right topology $\rho(E)$ of a Banach space $E$ consists of the collection of all seminorms $p$ such that there is a reflexive Banach space $Z$ and a bounded linear operator $T: E \rightarrow Z$ such that $p(x)=\|T(x)\|, x \in E$. This result is no longer true for non normable Fréchet spaces $E$. Indeed, there is a Fréchet Montel space $E$ which has the Banach space $\ell_{1}$ as a quotient; see e.g. Section 31.5 in [18]. Since $E$ is Fréchet Montel, it is reflexive and the Right topology on $E$ is the original Fréchet topology. Denote by $q: E \rightarrow \ell_{1}$ the quotient map. Clearly, $P(x):=\|q(x)\|$ is a continuous seminorm on $E$. If one can find a fundamental system of seminorms of the Right topology on $E$ as described above, then there are a reflexive Banach space $X$ and a continuous operator $T: E \rightarrow X$ such that $P(x) \leq\|T(x)\|$ for every $x \in E$. We may assume that $T$ has dense range, since every closed subspace of a reflexive Banach space is also reflexive. Define a map $S$ from $T(E) \subset X$ endowed with the norm of $X$ into $\ell_{1}$ as follows. We set $S(T(x)):=q(x), x \in E$. The map $S$ is well defined, for if $T(x)=T\left(x^{\prime}\right)$, then $\left\|q\left(x-x^{\prime}\right)\right\|=P\left(x-x^{\prime}\right) \leq\left\|T\left(x-x^{\prime}\right)\right\|=0$, hence $q(x)=q\left(x^{\prime}\right)$. Moreover $S$ is continuous, as $\|S(T(x))\|=\|q(x)\|=P(x) \leq\|T(x)\|$ for each $x \in E$. Using the density of $T(E)$ in $X$, we extend $S$ to a continuous linear operator 
$S: X \rightarrow \ell_{1}$. By the very definition, $S \circ T=q$. Now the surjectivity of $q$ implies that $S: X \rightarrow \ell_{1}$ is surjective. Apply the open mapping theorem to conclude that $\ell_{1}$ is isomorphic to a quotient of the reflexive space $X$. This is a contradiction, since every quotient of a reflexive Banach space is also reflexive.

Now we apply the results of Section 4 and Theorem 3.1 to get some consequences that can be seen as non-commutative vector valued extensions of Nikodym's theorem as it is explained in section 5 of [40]. See also the article [40] for a version of our next two theorems in the case of Banach valued operators.

Theorem 5.6 Let $A$ be a Banach space and let $E$ be a Fréchet space or a complete (DF)space. Let $\left(T_{n}\right)_{n}$ be a sequence of weakly compact operators from $A$ into $E$ such $\left(T_{n}^{\prime \prime} z\right)_{n}$ converges to 0 in $E$ for each $z \in A^{\prime \prime}$. Then

(1) If $\left(a_{j}\right)_{j} \subset A$ converges to 0 in the Right topology of $A$, then $\sup _{n \in \mathbb{N}} p\left(T_{n}\left(a_{j}\right)\right)$ converges to 0 as $j \rightarrow \infty$ for each continuous seminorm $p$ on $E$.

(2) If $\left(z_{j}\right)_{j}$ is a sequence in $A^{\prime \prime}$ converging to 0 in the topology $\mu\left(A^{\prime \prime}, A^{\prime}\right)$, then $\sup _{n \in \mathbb{N}} q\left(T_{n}^{\prime \prime}\left(z_{j}\right)\right)$ converges to 0 as $j \rightarrow \infty$ for each continuous seminorm $q$ on $\left(E^{\prime \prime}, \beta\left(E^{\prime \prime}, E^{\prime}\right)\right)$.

Proof. (1) The operator $\tilde{T}: A \rightarrow c_{0}(E), \tilde{T} a:=\left(T_{n} a\right)_{n}, a \in A$ is weakly compact by the extension of Ryan's result Corollary 4.7. Let $\left(a_{j}\right)_{j} \subset A$ be a sequence converging to 0 in the Right topology of $A$. Since $\tilde{T}$ is weakly compact, we can apply Theorem 5.3 to conclude that $\left(\tilde{T} a_{j}\right)_{j}$ converges to 0 in $c_{0}(E)$. This implies the conclusion using the form of the seminorms of $c_{0}(E)$.

(2) Let $\left(z_{j}\right)_{j}$ be a sequence in $A^{\prime \prime}$ converging to 0 in the topology $\mu\left(A^{\prime \prime}, A^{\prime}\right)$. Since $\tilde{T}$ is weakly compact, we can apply condition (2) in Theorem 5.3 to conclude that

$$
\tilde{T}^{\prime \prime}:\left(A^{\prime \prime}, \mu\left(A^{\prime \prime}, A^{\prime}\right)\right) \rightarrow\left(c_{0}(E)^{\prime \prime}, \beta\left(c_{0}(E)^{\prime \prime}, c_{0}(E)^{\prime}\right)\right)
$$

is continuous. By Proposition 4.1, $c_{0}(E)_{b}^{\prime \prime}=\ell_{\infty}\left(E_{b}^{\prime \prime}\right)$. Moreover, Corollary 4.7 implies $\tilde{T}^{\prime \prime} z=$ $\left(T_{n}^{\prime \prime} z\right)_{n}$ for each $z \in A^{\prime \prime}$. On account of the form of the seminorms of $\ell_{\infty}\left(E_{b}^{\prime \prime}\right)$, the conclusion follows.

Theorem 5.7 Let $A$ be a Banach space such that $\left(A^{\prime}, \sigma\left(A^{\prime}, A^{\prime \prime}\right)\right)$ is sequentially complete and let $E$ be a Fréchet space or a complete $(D F)$-space. Let $\left(T_{n}\right)_{n}$ be a sequence of weakly compact operators from $A$ into $E$ such that $\left(T_{n}^{\prime \prime} z\right)_{n}$ is a Cauchy sequence in $E$ for each $z \in A^{\prime \prime}$. Let $S a:=\lim T_{n} a$ for each $a \in A$. Then

(1) If $\left(a_{j}\right)_{j} \subset A$ converges to 0 in the Right topology of $A$, then $\sup _{n \in \mathbb{N}} p\left(\left(T_{n}-S\right)\left(a_{j}\right)\right)$ converges to 0 as $j \rightarrow \infty$ for each continuous seminorm $p$ on $E$.

(2) If $\left(z_{j}\right)_{j}$ is a sequence in $A^{\prime \prime}$ converging to 0 in the topology $\mu\left(A^{\prime \prime}, A^{\prime}\right)$, then $\sup _{n \in \mathbb{N}} q\left(\left(T_{n}^{\prime \prime}-\right.\right.$ $\left.\left.S^{\prime \prime}\right)\left(z_{j}\right)\right)$ converges to 0 as $j \rightarrow \infty$ for each continuous seminorm $q$ on $\left(E^{\prime \prime}, \beta\left(E^{\prime \prime}, E^{\prime}\right)\right)$.

Proof. By Theorem 3.1, $S$ is weakly compact and $S^{\prime \prime} z=\lim T_{n}^{\prime \prime} z$ in $E$ for each $z \in A^{\prime \prime}$. The conclusion now follows by applying Theorem 5.6 to the sequence of operators $\left(T_{n}-S\right)_{n}$.

We emphasize that this theorem would fail, in general, if $\left(A^{\prime}, \sigma\left(A^{\prime}, A^{\prime \prime}\right)\right)$ were not sequentially complete. See [33] and [42]. 


\section{Weakly compact operators from a $C^{*}$-algebra into a locally convex space}

We recall [34] the following result: Let $\psi \in A^{\prime}$ be a positive functional on a $C^{*}$-algebra $A$, and define

$$
p_{\psi}(a):=\psi\left(\left(a a^{*}+a^{*} a\right)^{1 / 2}\right), a \in A .
$$

Then $p_{\psi}$ is a seminorm on $A$. A positive functional $\psi \in A^{\prime}$ satisfying $\|\psi\|=1$ is called a state. The universal $\sigma$-strong* topology of a $C^{*}$-algebra $A$ is the topology induced by all seminorms $p_{\psi}$, where $\psi$ is a positive functional on $A$. It follows from a fundamental result of Akemann [1], see [34, Theorem III.5.7], that the restriction of the $\sigma$-strong* topology to the unit ball $A_{1}$ of $A$ coincides with the restriction of the Right topology $\rho(A)$ to $A_{1}$.

As in [41] by an orthogonal sequence in the $C^{*}$-algebra $A$ we mean a sequence $\left(a_{n}\right)_{n}$ of self adjoint elements of the closed unit ball of $A$ such that $a_{n} a_{m}=0$ whenever $n \neq m$.

The following omnibus Theorem 6.3 collects several characterizations of weakly compact operators from a $C^{*}$-algebra with values in a complete locally convex space. It is an extension of [41, Proposition 2.2] and its proof depends on deep characterizations of weak compactness in preduals of von Neumann algebras [2,34], and in duals of $C^{*}$-algebras [29]. We also use of methods due to Jarchow [15] and extend [15, Theorem 1.3]. Related results can be seen in Section 7. In the proof of Theorem 6.3 several lemmas are needed. The following result due to Akemann [1] (see [34, p.149]) is important.

Lemma 6.1 A subset $K \subset A^{\prime}$ is relatively $\sigma\left(A^{\prime}, A^{\prime \prime}\right)$-compact if and only if $K$ is bounded and there exists a state $\phi \in A^{\prime}$ such that for all $\varepsilon>0$ there exists $\delta>0$ such that for all $a \in A_{1}$ for which $p_{\phi}(a)<1$ then $|\psi(a)|<\varepsilon$ for all $\psi \in K$.

Lemma 6.2 $A$ bounded subset $K \subset A^{\prime}$ is relatively $\sigma\left(A^{\prime}, A^{\prime \prime}\right)$-compact if and only if there exist a state $\phi \in A^{\prime}$ and $\left.N:\right] 0, \infty[\rightarrow] 0, \infty[$ such that

$$
\sup _{\psi \in K}|\psi(a)| \leq N(\varepsilon) p_{\phi}(a)+\varepsilon\|a\|
$$

for all $a \in A$ and for all $\varepsilon>0$.

\section{Proof.}

Assume that such a state $\phi \in A^{\prime}$ can be found as in the assumption. Given $\varepsilon>0$, select $\delta:=\frac{\varepsilon}{2 N(\varepsilon / 2)}$. Suppose that $a \in A_{1}$ satisfies $p_{\phi}(a)<\delta$. For each $\psi \in K$ we have

$$
|\psi(a)| \leq N(\varepsilon / 2) p_{\phi}(a)+\frac{\varepsilon}{2}\|a\| \leq \varepsilon .
$$

Suppose now that $K$ is relatively $\sigma\left(A^{\prime}, A^{\prime \prime}\right)$-compact. By Lemma 6.1 , we find a state $\phi \in A^{\prime}$ such that for all $\varepsilon>0$ there exists $\delta>0$ such that, for all $a \in A_{1}, p_{\phi}(a) \leq \delta$ implies $\sup _{\psi \in K}|\psi(a)| \leq \varepsilon$. We define $N(\varepsilon):=\varepsilon / \min (1, \delta)$. Fix $a \in A, a \neq 0, \varepsilon>0$, and $\delta=\delta(\varepsilon)$. Set

$$
a^{\prime}:=\frac{1}{\max \left(\|a\|, \min (1, \delta)^{-1} p_{\phi}(a)\right)} a .
$$

Clearly $a^{\prime} \in A_{1}$ and $p_{\phi}\left(a^{\prime}\right) \leq \min (1, \delta) \leq \delta$. Consequently $\sup _{\psi \in K}\left|\psi\left(a^{\prime}\right)\right| \leq \varepsilon$. This yields 


$$
\begin{aligned}
& \sup _{\psi \in K}|\psi(a)| \leq \varepsilon \max \left(\|a\|, \min (1, \delta)^{-1} p_{\phi}(a)\right) \leq \\
& \leq \varepsilon\|a\|+\frac{\varepsilon}{\min (1, \delta)} p_{\phi}(a)=N(\varepsilon) p_{\phi}(a)+\varepsilon\|a\| .
\end{aligned}
$$

Theorem 6.3 Let $A$ be a $C^{*}$-algebra, $E$ a complete locally convex space and $T: A \rightarrow E$ a continuous linear operator. The following conditions are equivalent.

(1) $T$ is a weakly compact operator.

(2) $T$ is pseudo weakly compact operator.

(3) If $\left(a_{n}\right)_{n}$ is an orthogonal sequence in $A$, then $\left(T a_{n}\right)_{n}$ converges to 0 in $E$.

(4) For every bounded universal strong $*$-null net $\left(a_{\lambda}\right)_{\lambda}$ in $A$ we have $\left(T\left(a_{\lambda}\right)\right)_{\lambda}$ converges to 0 in $E$.

(5) If $\left(a_{n}\right)_{n}$ is a sequence in $A$ which is convergent in the universal $\sigma$-strong* topology, then $\left(T a_{n}\right)_{n}$ converges in $E$.

(6) $T:(A, \rho(A)) \rightarrow E$ is continuous.

(7) For each $q \in \operatorname{cs}(E)$ there exist a state $\phi_{q} \in A^{\prime}$ and $\left.N_{q}:\right] 0, \infty[\rightarrow] 0, \infty[$ such that

$$
q(T a) \leq N_{q}(\varepsilon) p_{\phi_{q}}(a)+\varepsilon\|a\| .
$$

Proof. By Theorem 5.3 conditions (1) and (6) are equivalent. By the very definition condition (6) implies (2). We show that (2) implies (3). Let $\left(a_{n}\right)_{n}$ be an orthogonal sequence in $A$. As the proof of [41, Proposition 2.2], we conclude that $\left(a_{n}\right)_{n}$ converges to zero in the universal $\sigma$ strong* topology. A result of Akemann, see [34, Theorem III.5.7], assures that the restriction of the $\sigma$-strong* topology to the unit ball $A_{1}$ of $A$ coincides with the restriction of the Right topology $\rho(A)$ to $A_{1}$. Since $T:(A, \rho(A)) \rightarrow E$ is sequentially continuous by condition (2), we conclude that $\left(T a_{n}\right)_{n}$ converges to 0 in $E$. To prove that (3) implies (1), it is enough to show that $\pi_{q} \circ T: A \rightarrow E_{q}$ is weakly compact for each continuous seminorm $q \in \operatorname{cs}(E)$. We can apply (3) to conclude that $\lim _{n}\left\|T a_{n}\right\|_{E_{q}}=0$ for each orthogonal sequence $\left(a_{n}\right)_{n}$ in $A$. The conclusion follows from [41, Proposition2.2]. The implication needed at this point is based on a very deep theorem due to Pfitzner [29]. Clearly (4) implies (5), which is equivalent to (2), since the restriction of the $\sigma$-strong* topology to the unit ball $A_{1}$ of $A$ coincides with the restriction of the Right topology $\rho(A)$ to $A_{1}$. The same fact permits us to conclude (4) from (6).

It remains to show that $(7)$ is equivalent to the other six conditions. First we show that (7) implies (4). Let $\left(a_{\lambda}\right)_{\lambda}$ be a bounded net in $A$ which converges to 0 in the universal strong *-topology of $A$. Fix $q \in \operatorname{cs}(E)$. We apply (7) to find a state $\phi_{q} \in A^{\prime}$ and $\left.N_{q}:\right] 0, \infty[\rightarrow] 0, \infty[$ such that, for all $\varepsilon>0$ and for all $\lambda$

$$
q\left(T\left(a_{\lambda}\right)\right) \leq N_{q}(\varepsilon) p_{\phi_{q}}\left(a_{\lambda}\right)+\varepsilon\left\|a_{\lambda}\right\| .
$$


since the net is bounded, there is $M>0$ such that $\left\|a_{\lambda}\right\| \leq M$ for all $\lambda$. By [34, 2.5], $\left(\phi_{q}\left(a_{\lambda} a_{\lambda}^{*}\right)\right)_{\lambda}$ tends to 0 and $\left(\phi_{q}\left(a_{\lambda}^{*} a_{\lambda}\right)\right)_{\lambda}$ tends to 0 . This yields that $\left.\left(p_{\phi_{q}}\left(a_{\lambda}\right)\right)_{\lambda}\right)$ tends to 0 . Given $\varepsilon>0$, select $\lambda_{0}$ such that $p_{\phi_{q}}\left(a_{\lambda}\right)<\varepsilon /\left(2 N_{q}(\varepsilon / 2 M)\right)$ whenever $\lambda>\lambda_{0}$. For each $\lambda>\lambda_{0}$ we have

$$
\begin{gathered}
q\left(T\left(a_{\lambda}\right)\right) \leq N_{q}(\varepsilon / 2 M) p_{\phi_{q}}\left(a_{\lambda}\right)+\frac{\varepsilon}{2 M}\left\|a_{\lambda}\right\| \leq \\
\leq N_{q}(\varepsilon / 2 M) \frac{\varepsilon}{2 N_{q}(\varepsilon / 2 M)}+\frac{\varepsilon}{2 M} M=\varepsilon,
\end{gathered}
$$

and condition (4) follows.

Now we show that condition (1) implies condition (7). By Grothendieck's extension of Gantmacher's theorem, $T \in L(A, E)$ is weakly compact if and only if, for each $q \in \operatorname{cs}(E)$, $T^{t}\left(U_{q}^{\circ}\right)$ is a $\sigma\left(A^{\prime}, A^{\prime \prime}\right)$-compact subset of $A^{\prime}$. By Lemma 6.2 this is in turn equivalent to show that the following condition holds: for each $q \in \operatorname{cs}(E)$ there exists $\phi_{q} \in A^{\prime}$, a state, such that there exists $N:] 0, \infty[\rightarrow] 0, \infty[$ such that

$$
\sup _{\psi \in T^{t}\left(U_{q}^{\circ}\right)}|\psi(a)| \leq N(\varepsilon) p_{\phi_{q}}(a)+\varepsilon\|a\|
$$

for all $\varepsilon>0$ and $a \in A$. Since

$$
\sup _{\psi \in T^{t}\left(U_{q}^{\circ}\right)}=\sup _{u \in U_{q}^{\circ}}|u \circ T(a)|=q(T(a)),
$$

the conclusion follows.

In case $E$ is a Fréchet space, the state $\phi$ in condition (7) of Theorem 6.3 does not depend on the seminorm $q$.

Proposition 6.4 Let $E$ be a $C^{*}$-algebra and let $E$ be a Fréchet space. $T \in L(A, E)$ is weakly compact if and only if there exist a state $\phi \in A^{\prime}$ and $\left.N:\right] 0, \infty[\rightarrow] 0, \infty[$ such that for each $q \in \operatorname{cs}(E)$ there exists $\lambda_{q}>0$ for which

$$
q(T a) \leq N_{q}(\varepsilon) p_{\phi_{q}}(a)+\lambda_{q} \varepsilon\|a\|
$$

for all $a \in A$ and for all $\varepsilon>0$.

Proof. We only have to show that an operator $T$ which is weakly compact satisfies the assumption. Let $T \in L(A, E)$ weakly compact. Since $E$ is Fréchet, we find $B \in \mathcal{B}(E)$ such that $T\left(A_{1}\right)$ is contained in $B$ and it is relatively weakly compact in $E_{B}$. The map $T_{0}:=T: A \rightarrow E_{B}$ is continuous and weakly compact. By [15, Theorem 1.3] there exist $\phi \in A^{\prime}$ a state and $\left.N:\right] 0, \infty[\rightarrow] 0, \infty[$ such that

$$
\left\|T_{0}(a)\right\|_{E_{B}} \leq N(\varepsilon) p_{\phi}(a)+\varepsilon\|a\| \forall a \in A, \varepsilon>0 .
$$

Given $q \in \operatorname{cs}(E)$, since $B$ is bounded, there is $\lambda_{q}>0$ with $\sup _{x \in B} q(x) \leq \lambda_{q}$, thus $q(z) \leq$ $\lambda_{q}\|z\|_{E_{B}}$ for each $z \in E_{B}$. This implies, for $a \in A$

$$
q(T(a))=q\left(T_{0}(a)\right) \leq \lambda_{q} N(\varepsilon) p_{\phi}(a)+\lambda_{q} \varepsilon\|a\| .
$$




\section{Weak E-valued integral}

The purpose of this section is to present a locally convex extension of the main result of Wright [38]. It emphasizes the fact that weakly compact operators on a $C^{*}$-algebra constitute the natural non-commutative extensions of vector valued measures. Theorem 7.4 extends theorems of Lewis [19] and Panchapagesan [23, 24] to the non-commutative setting and of Wright's theorem for the case of operators with values in a locally convex space.

For a $C^{*}$-algebra $A$, we denote by $A_{s a}$ the set of self adjoint elements in $A$, and $A^{\sigma}$ stands for the smallest subspace of $A^{\prime \prime}$ containing $A$ with the property that whenever $\left(b_{n}\right)_{n}$ is a monotonic sequence $\left(A^{\sigma}\right)_{s a}$ with limit $b$ in the weak operator topology of $A^{\prime \prime}$ (or in the topology $\left.\sigma\left(A^{\prime \prime}, A^{\prime}\right)\right)$, then $b \in A^{\sigma}$. By a fundamental theorem of Pedersen [25], $A^{\sigma}$ is a $C^{*}$-subalgebra of $A^{\prime \prime}$ and it is called the Baire *-envelope of $A$ or the Pedersen envelope of $A$.

Definition $7.1 \tilde{T}: A^{\sigma} \rightarrow E$ is a weak E-valued integral for $A$ if it is continuous and for all $\left(b_{n}\right)_{n}$ monotonic sequence of self adjoint elements in $A^{\sigma}$ such that $b_{n}$ tends to $b$ for the $\sigma\left(A^{\sigma}, A^{\prime}\right)$-topology then $\tilde{T}\left(b_{n}\right)$ tends to $\tilde{T}(b)$ for the $\sigma\left(E, E^{\prime}\right)$-topology.

If $\tilde{T}: A^{\sigma} \rightarrow E$ is a weak $E$-valued integral for $A$, then $T:=\left.\tilde{T}\right|_{A}$ is a continuous linear operator $T: A \rightarrow E$ and $T^{\prime \prime}: A^{\prime \prime} \rightarrow E^{\prime \prime}$ is well-defined and continuous.

Lemma 7.2 Let $\tilde{T}: A^{\sigma} \rightarrow E$ be a weak E-valued integral for $A$ and let $T:=\left.\tilde{T}\right|_{A}$ be the restriction of $\tilde{T}$ to $A$. Then $\tilde{T}=\left.T^{\prime \prime}\right|_{A^{\sigma}}$.

Proof. As in [38, Lemma 2.2], let $V:=\left\{b \in A^{\sigma} \mid \tilde{T}(b)=T^{\prime \prime}(b)\right\} . V$ is a closed subspace of $A^{\prime \prime}$ which contains $A$. Let $\left(b_{n}\right)_{n}$ be a monotonic sequence of self adjoint elements in $V$ with limit $b$ in $\sigma\left(A^{\prime \prime}, A^{\prime}\right)$. For each $u \in E^{\prime}$,

$$
\langle u, \tilde{T}(b)\rangle=\lim _{n \rightarrow \infty}\left\langle u, T^{\prime \prime}\left(b_{n}\right)\right\rangle=\lim _{n \rightarrow \infty}\left\langle T^{\prime}(u), b_{n}\right\rangle=\left\langle T^{\prime}(u), b\right\rangle=\left\langle u, T^{\prime \prime}(b)\right\rangle .
$$

This implies $b \in V$. By the minimality of $A^{\sigma}, V=A^{\sigma}$.

Lemma 7.3 Let $\tilde{T}: A^{\sigma} \rightarrow E$ be a weak E-valued integral for $A$. If $\left(p_{n}\right)_{n}$ is a decreasing sequence of projections in $A^{\sigma}$ with infimum 0 , then $\lim _{n \rightarrow \infty} q\left(\tilde{T}\left(p_{n}\right)\right)=0$ for each $q \in \operatorname{cs}(E)$.

Proof. Fix $q \in \operatorname{cs}(E)$. The continuous linear operator $\pi_{q} \circ T: A^{\sigma} \rightarrow E_{q}$ is a weak valued integral in the sense of Wright [38, p.126]. By Lemma 2.2 in [38],

$$
\left\|\pi_{q} \circ \tilde{T}\left(p_{n}\right)\right\|_{E_{q}} \rightarrow 0 \quad \text { as } \quad n \rightarrow \infty .
$$

Since $q(z)=\left\|\pi_{q}(z)\right\|_{E_{q}}$ for each $z \in E$, we have

$$
\lim _{n \rightarrow \infty} q\left(\tilde{T}\left(p_{n}\right)\right)=0
$$

Theorem 7.4 A continuous operator $T: A \rightarrow E$ is weakly compact if and only if there is a weak E-valued integral $\tilde{T}: A^{\sigma} \rightarrow E$ for $A$ whose restriction to $A$ coincides with $T$. 
Proof. If $T \in L(A, E)$ is weakly compact, Grothendiek's extension of Gantmacher's theorem $[18,42.2 .(1)]$ ensures that $T^{\prime \prime}\left(A^{\prime \prime}\right) \subset E$ and the operator $T^{\prime}:\left(A^{\prime \prime}, \sigma\left(A^{\prime \prime}, A^{\prime}\right)\right) \rightarrow\left(E, \sigma\left(E, E^{\prime}\right)\right)$ is continuous. This implies that $\tilde{T}:=\left.T^{\prime \prime}\right|_{A^{\sigma}}$ is a weak $E$-valued integral which extends $T$.

To show that the converse is also true we assume $T=\left.\tilde{T}\right|_{A}$ for a weak $E$-valued integral $\tilde{T}: A^{\sigma} \rightarrow E$. By Grothendieck's extension of Gantmacher's theorem we must show that for each $q \in \operatorname{cs}(E), T^{\prime}\left(U_{q}^{\circ}\right)$ is a relatively $\sigma\left(A^{\prime}, A^{\prime \prime}\right)$-compact subset of $A^{\prime}$.

$$
T^{\prime}\left(U_{q}^{\circ}\right)=\left\{u \circ T\left|u \in E^{\prime},\right| u(x) \mid \leq q(x) \text { for all } x \in E\right\} .
$$

Clearly $T^{\prime}\left(U_{q}^{\circ}\right)$ is bounded in $A^{\prime}$, since $T$ is continuous. Suppose first that $A$ is separable. By [38, Lemma 2.1], it is enough to show that if $\left(p_{n}\right)_{n}$ is a monotone decreasing sequence of projections in $A^{\sigma}$ with infimum 0 , then $\lim _{n \rightarrow \infty} \mu\left(p_{n}\right)=0$ uniformly on $\mu \in T^{\prime}\left(U_{q}^{\circ}\right)$.

By Lemma 7.3 , if $\left(p_{n}\right)$ is a monotone decreasing sequence of projections in $A^{\sigma}$ with $\inf _{n} p_{n}=0$, then $\lim _{n \rightarrow \infty} q\left(\tilde{T}\left(p_{n}\right)\right)=0$. But

$$
q\left(\tilde{T}\left(p_{n}\right)\right)=\sup _{u \in U_{q}^{\circ}}\left|u\left(\tilde{T}\left(p_{n}\right)\right)\right| .
$$

On the other hand, we can apply Lemma 7.2 to conclude, for $u \in U_{q}^{\circ}$

$$
u\left(\tilde{T}\left(p_{n}\right)\right)=u\left(T^{\prime \prime}\left(p_{n}\right)\right)=\left(T^{\prime}(u)\right)\left(p_{n}\right) .
$$

Therefore

$$
\lim _{n \rightarrow \infty} \sup _{u \in U_{q}^{\circ}}\left|\left(T^{\prime}(u)\right)\left(p_{n}\right)\right|=0,
$$

and $\lim _{n \rightarrow \infty} \mu\left(p_{n}\right)=0$ uniformly for $\mu \in T^{\prime}\left(U_{q}^{\circ}\right)$. Accordingly, if $A$ is separable, $T: A \rightarrow E$ is weakly compact. The general case now follows from [2, Theorem 3.1] or by the argument at the end of the proof of [38, Theorem 2.3].

The commutative case of our next result is [24, Theorem 13]. The non-commutative case for a Banach space $E$ is due to Akemann, Dodds and Gamlen [2]

Corollary 7.5 If $A$ be a $C^{*}$ algebra and $E$ is a complete locally convex space which contains no copy of $c_{0}$, then every $T \in L(A, E)$ is weakly compact.

Proof. Let $V:=\left\{a \in A^{\prime \prime} \mid T^{\prime \prime}(a) \in E\right\}$. Then $V$ is a closed subspace of $A^{\prime \prime}$ containing $A$. Let $\left(b_{n}\right)_{n}$ be a monotonic and bounded sequence of self-adjoint elements in $V$ such that $b_{n}$ tends to $b$ in $\sigma\left(A^{\prime \prime}, A^{\prime}\right)$. For each positive element $f \in A^{\prime}$ we have

$$
\sum_{n=1}^{\infty}\left|f\left(b_{n+1}-b_{n}\right)\right|<\infty \text {. }
$$

If $u \in E^{\prime}$ then $T^{\prime}(u) \in E^{\prime}$ and we can write $T^{\prime}(u)=f_{1}-f_{2}+i\left(f_{3}-f_{4}\right), f_{i} \in A^{\prime}$ positive. Hence

$$
\sum_{n=1}^{\infty}\left|\left\langle b_{n+1}-b_{n}, T^{\prime}(u)\right\rangle\right|=\sum_{n=1}^{\infty}\left|\left\langle T^{\prime \prime}\left(b_{n+1}-b_{n}\right), u\right\rangle\right|<\infty .
$$

and the series $\sum_{n=1}^{\infty} T^{\prime \prime}\left(b_{n+1}-b_{n}\right)$ is weakly unconditionally Cauchy. By a result of Tumarkin [35] (see also $[16,20]), \sum_{n=1}^{\infty} T^{\prime \prime}\left(b_{n+1}-b_{n}\right)=x \in E$, the convergence of the series being for 
the topology of $E$. Since $b_{n}$ tends to $b$ in $\sigma\left(A^{\prime \prime}, A^{\prime}\right)$, it follows that $T^{\prime \prime}\left(b_{n}\right)$ tends to $T^{\prime \prime}(b)$ in $\sigma\left(E^{\prime \prime}, E^{\prime}\right)$. Hence we can write

$$
T^{\prime \prime}(b)=T^{\prime \prime}\left(b_{1}\right)+\sum_{n=1}^{\infty} T^{\prime \prime}\left(b_{n+1}-b_{n}\right),
$$

the convergence of this series being in $\sigma\left(A^{\prime \prime}, A^{\prime}\right)$. However $\sum_{n=1}^{\infty} T^{\prime \prime}\left(b_{n+1}-b_{n}\right)=x \in E$ converges in $E$. This yields

$$
T^{\prime \prime}(b)=T^{\prime \prime}\left(b_{1}\right)+x \in E .
$$

Hence $b \in V$ and this implies $A^{\sigma} \subset V$, and $\tilde{T}:=\left.T^{\prime \prime}\right|_{A^{\sigma}}$ is a weak $E$-valued integral for $A$. Now $T$ is weakly compact by Theorem 7.4.

\section{References}

[1] C.A. Akemann: The dual space of an operator algebra, Trans. Amer. Math. Soc. 126 (1967), 286-302.

[2] C.A. Akemann, P.G. Dodds, J.L.B. Gamlen: Weak compactness in the dual of a $C^{*}$-algebra, J. Funct. Anal. 10 (1972), 446-450.

[3] K.D. Bierstedt, J. Bonet: Some aspects of the modern theory of Fréchet spaces, Rev. R. Acad. Ci. Serie A Mat. 97 (2003), 159-188.

[4] J.K. Brooks, R.S. Jewett: On finitely additive vector measures, Proc. nat. acad. Sciences 67 (1970) 1294-1298.

[5] J.K. Brooks, K. Saitô, J.D.M. Wright: Operator algebras and a theorem of Dieudonné, Rend. Circolo Mat. Palermo 52 (2003), 5-14.

[6] J.K. Brooks, K. SAitô, J.D.M. Wright: When absolute continuity on $C^{*}$-algebras is automatically uniform, Quaterly J. Math. 55 (2004), 31-40.

[7] J.K. Brooks, J.D.M. Wright: Representing Yosida-Hewitt decomposition for classical and non-commutative vector measures, Expo. Math. 19 (2001), 373-383.

[8] J.K. Brooks, J.D.M. Wright: Convergence in the dual of a $\sigma$-complete $C^{*}$-algebra, J. Math. Anal. Appl. 294 (2004), 141-146.

[9] J.K. Brooks, K. Saitô, J.D.M. Wright: Operators on $\sigma$-complete $C^{*}$-algebras, Quaterly J. Math. 56 (2005), 301-310.

[10] J. DíAz, P. Domański: Reflexive operators with domain in Köthe spaces, Manuscripta Math. 97 (1998), 189-204.

[11] J. Dieudonné: Sur la convergence des suites des mesures de Radon, An. Acad. Brasil. Ci. 23 (1951), 277-282.

[12] A. Grothendieck: Sur les applications linéaries faiblement compactes d'espaces du type $C(K)$, Canad. J. Math. 5 (1953), 129-173. 
[13] A. Grothendieck: Topological Vector Spaces, New York, 1973.

[14] H. JARChOw: Locally Convex Spaces; Math. Leitfäden, B.G. Teubner, Stuttgart (1981).

[15] H. Jarchow: On weakly compact operators on $C^{*}$-algebras, Math. Ann. 273 (1986), 341-343.

[16] N. J. Kalton: Exhaustive operators and vector measures, Proc. Edinburgh Math. Soc. $19(1974 / 75), 291-300$.

[17] I. KLUvanEK: Fourier transforms of vector-valued functions and measures, Studia Math. 37 (1970), 1-12.

[18] G. KӧтнE: Topological Vector Spaces I and II, Springer Verlag, Berlin-Heidelberg-New York, 1969 and 1979.

[19] D.R. LewIS: Integration with respect to vector measures, Pacific J. Math. 33 (1970), $157-165$.

[20] L. R. Li, Q. Y. Bu: Locally convex spaces containing no copy of $c_{0}$, J. Math. Ann. Appl. 172 (1993), 205-211.

[21] R. Meise, D. Vogt: Introduction to Functional Analysis, Clarendon Press, Oxford, 1997.

[22] G.J. Murphy: $C^{*}$-algebras and Operator Theory, Academic Press, Boston, 1990.

[23] T. V. Panchapagesan: A simple proof of the Grothendieck theorem on the Dieudonné property of $C_{0}(T)$, Proc. Amer. Math. Soc. 129 (2001), 823-831.

[24] T.V. Panchapagesan: Characterization of weakly compact operators on $C_{0}(T)$, Trans. Amer. Math. Soc. 350 (1998), 4845-4867.

[25] G.K. Pedersen: $C^{*}$-algebras and their automorphism groups, London-New York, Academic Press, 1979.

[26] A.M. Peralta: Topological characterization of weakly compact operators revisited, Extracta Math. 22 (2007), 215-223.

[27] A.M. Peralta, I. Villanueva, J.D.M. Wright, K. Ylinen: Topological characterization of weakly compact operators, J. Math. Anal. Appl. 325 (2007), 968-974.

[28] P. Pérez Carreras and J. Bonet: Barrelled Locally Convex Spaces, Vol. 131, NorthHolland, Amsterdam, 1987.

[29] H. Pfitzner: Weak compactness in the dual of a $C^{*}$-algebra is determined commutatively, Math. Anal. 293 (1994), 349-371.

[30] A. Pietsch: Nuclear Locally Convex Spaces, Springer, New York, 1972.

[31] A.P. Robertson, W. Robertson: Topological Vector Spaces, Cambridge University Press, Cambridge, 1964.

[32] R.A. Ryan: Dunford-Pettis properties, Bull. Polish Acad. Sci. Math. 27 (1979), 373-379. 
[33] K. Saitô, J.D.M. Wright: Extending a result of Ryan on weakly compact operators, Proc. Edinb. Math. Soc. 49 (2006), 427-433.

[34] M. Takesaki: Theory of Operator Algebras I, Springer, New York, 1979.

[35] Ju. B. Tumarkin: On locally convex spaces with basis, Dokl. Akad. Nauk SSSR 195 (1970), 1672-1675.

[36] Y.-C. Wong, N.-C. Wong: Topologies and bornologies determined by operator ideals, Math. Ann. 282 (1988), 587-614.

[37] N.-C. Wong: Topologies and bornologies determined by operator ideals II, Studia Mathematica 111 (1994), 153-162.

[38] J.D.M. Wright: Operators from $C^{*}$-algebras to Banach spaces, Math. Z. 172 (1980), 123-129.

[39] J.D.M. Wright: Every monotone $\sigma$-complete $C^{*}$-algebra is the quotient of its Baire* envolpe by a two sided $\sigma$-ideal, J. London Math. Soc. 6 (1973), 210-214.

[40] J.D.M. Wright: Right topology for Banach spaces and weak compactness, Atti. Semin. Mat. Fis. Univ. Modena Reggio Emilia 55 (2007), 153-163.

[41] J.D.M. Wright, K. Ylinen: Multilinear maps on products of operator algebras, J. Math. Anal. Appl. 292 (2004), 558-570.

[42] K. Ylinen: Weakly compact operators into sequence spaces: a counterexample, Proc. Amer. Math. Soc. 133 (2005), 1423-1425.

\section{Authors' addresses:}

José Bonet

Instituto Universitario de Matemática

Pura y Aplicada IUMPA,

Universidad Politécnica de Valencia,

E-46071 Valencia, Spain

E-mail: jbonet@mat.upv.es
J.D.M. Wright

Mathematical Sciences,

Kings College,

University of Aberdeen,

Aberdeen AB24 3UE, Great Britain

E-mail: j.d.m.wright@abdn.ac.uk 